\title{
Johne's Disease (Paratuberculosis) in a Crossbred Cow: A Case Report
}

\author{
A. Karthikeyan ${ }^{1 *}$, L. Gunaseelan ${ }^{1}$, K. Porteen ${ }^{1}$, \\ B. Samuel Masilamoni Ronald ${ }^{2}$ and S. Pavithra ${ }^{3}$ \\ ${ }^{1}$ Department of Veterinary Public Health and Epidemiology, Madras Veterinary College, \\ Chennai-600007, Tamil Nadu, India \\ ${ }^{2}$ Department of Veterinary Microbiology, Madras Veterinary College, \\ Chennai-600007, Tamil Nadu, India \\ ${ }^{3}$ Division of Veterinary Pharmacology and Toxicology, Indian Veterinary Research Institute, \\ Bareilly- 243 12, Uttar Pradesh, India \\ *Corresponding author
}

\section{A B S T R A C T}

\section{Keywords}

Johne's disease, Mycobacterium avium subsp. paratuberculosis, ZN staining and IS900 PCR

Article Info

\section{Accepted:}

22 December 2019

Available Online:

20 January 2020
Johne's disease (Paratuberculosis), a debilitating chronic granulomatous enteritis of domesticated and wild ruminants that causes huge economic and production losses to the dairy farmers. A six-year-old Jersey crossbred cow was presented with a history of chronic diarrhoea, gradual weight loss, reduction in milk yield and poor response to therapy. Based on history, clinical examination, ZiehlNeelsen staining and IS900 polymerase chain reaction of the faeces the case was diagnosed as Johne's disease. It warrants strict implementation of control measures to put off further spread Johne's disease in dairy animals.

\section{Introduction}

Johne's disease (Paratuberculosis) is chronic infectious granulomatous enteritis of domestic as well as wild ruminants caused by Mycobacterium avium subsp. paratuberculosis (Windsor and Whittington, 2010; Sweeney, 2011; Momotani et al., 2012). It is characterized by a long incubation period followed by persistent diarrhoea, progressive emaciation, decreased milk production, oedema, anaemia and death (Harris and Barletta, 2001). Johne's disease is still incurable due to poor response of MAP organisms to anti-mycobacterial therapy as well as other treatments (Sharif et al., 2013). The present case deals with a paratuberculosis (Johne's disease) in a crossbred cow. 


\section{Case history and Clinical examination}

A six-year-old Jersey crossbred cow from private dairy farm, Madhavaram reported to Department of Veterinary Public Health and Epidemiology, Madras Veterinary College, Chennai with the history of gradual weight loss and persistent diarrhoea for the past one month that is neither offensive nor blood stained. The animal became progressively weaker and emaciated despite having good appetite and feeding. The milk yield also reduced from eight litres/day to two litres/day. The animal purchased four months back from the local sandy and maintained under the semi-intensive system of management. The animal treated by a practising veterinarian and non-responsive to the antimicrobial and anthelmintic therapy.

Clinical examination revealed emaciation with the evidence of watery diarrhoea in the animal (Figure 1). A large sized oedema also observed in the sub-mandibular region. The cardinal signs such as temperature, heart rate and respiration rate were all within normal range. Rectal palpation showed that animal had soft watery, light brown faeces and mild corrugation of the rectal wall also felt. Dung and rectal pinch samples were collected and transported to the laboratory under refrigerated conditions.

\section{Laboratory diagnosis}

About $1.5 \mathrm{gm}$ of dung sample was mixed thoroughly with $10 \mathrm{ml}$ of $0.90 \%$ sterile saline in a sterile tube and the tube was shaken vigorously to mix the suspension. Then suspension was allowed to settle for 30 minutes. All supernatant solution was transferred to another sterile tube and centrifuged at $3200 \mathrm{rpm}$ for $30 \mathrm{~min}$ at room temperature. Then supernatant was discarded without dislodging the pellet, and the pellet was processed for smear preparation and
DNA isolation (Whipple et al., 1991). Furthermore, faecal sample also subjected to centrifugal sedimentation to rule out the presence of parasitic ova/ oocyst.

The smears made from sediment pellet and rectal pinch were stained with commercial Ziehl-Neelsen staining kit (Hi-Media, Mumbai) as per the manufacturer's instructions. The extracted DNA was amplified by Polymerase Chain reaction (PCR) using specific IS900 primers (Forward: 5'- CGT CGT TAA TAA CAA TGC AG 3'and Reverse: 5'- GGC CGT GCG TTA GGC TTC GA -3') (Giese and Ahrens, 2000). Presence specific PCR amplicons at $279 \mathrm{bp}$ considered as positive for IS900 segment of MAP, which indicates Johne's disease infection.

\section{Results and Discussion}

Examination of Ziehl-Neelsen stained rectal pinch and faecal smears revealed the clusters of acid-fast rods morphologically resembling M. avium subsp. paratuberculosis (Figure 2). Further examination of dung samples does not revealed the presence of any parasitic ova/cyst. The PCR showed an amplification product of $279 \mathrm{bp}$ which corresponds to $M$. avium subsp. paratuberculosis (Figure 3). Based on history, clinical symptoms and laboratory investigation the case was diagnosed as Johne's disease (paratuberculosis).

Johne's disease (paratuberculosis) is a is a chronic wasting disease of dairy cattle that causes huge economic losses globally among dairy farmers primarily via reduction in milk production, infertility, increased incidence of mastitis and shorter life expectancy of animals (Ott et al., 1999; Lombard, 2011). A wide array of diagnostic tests including single intradermal test, Ziehl-Neelsen staining, Cultural isolation, ELISA and Polymerase 
chain reaction were used for the diagnosis of Johne's disease in dairy animals. As none of the test is $100 \%$ sensitive, a battery of diagnostic tests has been recommended to increase the accuracy of MAP diagnosis
(Stevenson, 2010). Further, treatment against paratuberculosis requires prolonged medication as a palliative therapy and recurrence of the disease may occur in the farm (St-Jean, 1996; Sharif et al., 2013).

Fig.1 Emaciation and external appearance of cow

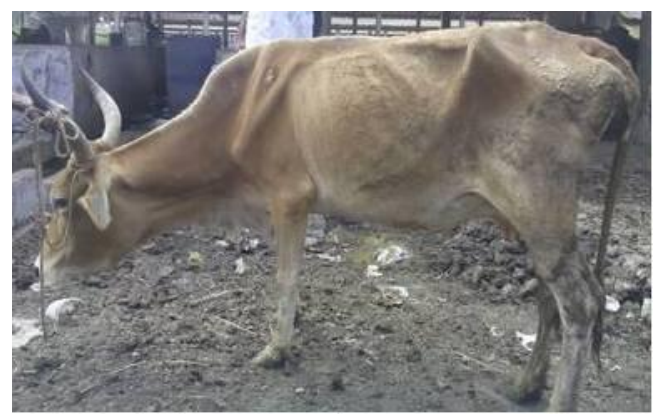

Fig.2 Clusters of MAP bacilli in dung smear

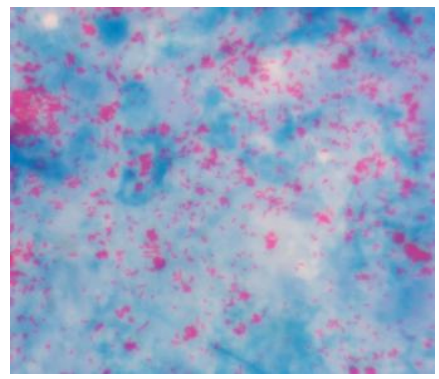

Fig.3 Agarose gel showing amplicons for IS900 PCR of MAP in suspected dung sample (Lane 1:

DNA Marker (100bp), Lane 2: Positive control, Lane 3: Negative control,

Lane 4: Suspected sample)

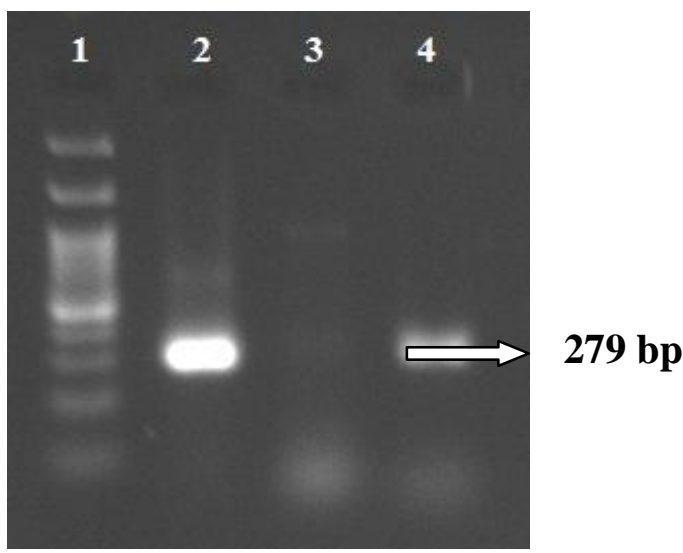


The present case revealed the presence of paratuberculosis in the dairy population of Tamil Nadu. It warrants comprehensive epidemiological studies and strict implementation of suitable control measures to combat against the scourge of paratuberculosis in animals.

\section{References}

Corbett, C. S., J. Buck, K. Orsel and Barkema, H. W. 2017. Fecal shedding and tissue infections demonstrate transmission of Mycobacterium avium subsp. paratuberculosis in grouphoused dairy calves. Veterinary Research. 48(1): 27.

Giese, S. B., and Ahrens, P. 2000. Detection of Mycobacterium avium subsp. paratuberculosis in milk from clinically affected cows by PCR and culture. Veterinary Microbiology. 77: 291-297.

Harris, N. B., and Barletta, R. G. 2001. Mycobacterium avium subsp. paratuberculosis in veterinary medicine. Clinical Microbiology Reviews. 14(3): 489-512.

Lombard, J. E. 2011. Epidemiology and economics of paratuberculosis. Veterinary Clinics of North America: Food Animal Practice. 2011; 27(3): 525-535.

Momotani, E., N. M. Romona, K. Yoshihara, Y. Momotani, M. Hori, H. Ozaki, S. Eda and Ikegami, M. 2012. Molecular pathogenesis of bovine paratuberculosis and human inflammatory bowel diseases. Veterinary Immunology and Immunopathology. 148: 55-68.

Ott, S. L., S. J. Wells and Wagner, B. A. 1999. Herd-level economic losses associated with Johne's disease on US dairy operations. Preventive Veterinary Medicine. 40(3): 179-192.

Sharif, M. A., M. E. Farhat, E. S. Kraim, N. A. Altrabulsi, A. M. Kammon, A.S. Dayhum and Eldaghayes, I. M. 2013. Ovine paratuberculosis: a confirmed case of Johne's disease in Libya. Open Veterinary Journal. 3(2): 131-134.

Stevenson, K., 2010. Diagnosis of Johne's disease: current limitations and prospects. Cattle Practice. 18: 104-109.

St-Jean, G., 1996. Treatment of clinical paratuberculosis in cattle. Veterinary Clinics of North America: Food Animal Practice. 12(2): 417-430.

Sweeney, R. W., 2011. Pathogenesis of paratuberculosis. Veterinary Clinics of North America: Food Animal Practice. 27(3): 537-546.

Whipple, D. L., D. R. Callihan and Jarnagin, J. L. 1991. Cultivation of Mycobacterium paratuberculosis from bovine fecal specimens and a suggested standardized procedure. Journal of Veterinary Diagnostic Investigation. 3(4): 368-373.

Windsor, P. A., and Whittington, R. J. 2010. Evidence for age susceptibility of cattle to Johne's disease. Veterinary Journal. 184(1): 37-44.

\section{How to cite this article:}

Karthikeyan, A., L. Gunaseelan, K. Porteen, B. Samuel Masilamoni Ronald and Pavithra, S. 2020. Johne's Disease (Paratuberculosis) in a Crossbred Cow: A Case Report. Int.J.Curr.Microbiol.App.Sci. 9(01): 2264-2267. doi: https://doi.org/10.20546/ijcmas.2020.901.256 